\title{
I cattolici e la politica fra le due guerre. Dalla lotta fra popolarismo e clerico-fascismo alla nascita della Democrazia cristiana
}

Giuseppe Matulli

\section{Premessa}

Appena conclusa la Grande guerra, partono le iniziative guidate da don Luigi Sturzo volte alla costituzione del Partito popolare italiano, che sarà fondato a Roma il 18 gennaio 1919. In previsione della sua nascita, il 22 dicembre del 1918 , Antonio Gramsci ne aveva già parlato come del «fatto più grande dopo il Risorgimento», mentre un trentennio più tardi lo storico Federico Chabod lo definirà «il fatto più straordinario della storia d'Italia del XX secolo», segno del definitivo superamento dell'opposizione allo Stato unitario da parte dei cattolici, che, dopo aver partecipato alla guerra, vi entrano adesso attraverso il $\mathrm{Ppi}^{1}$. A fondamento di valutazioni così impegnative, vi è la percezione di una sorta di discontinuità politica e culturale del nuovo evento rispetto alle vicende che come vedremo - avevano segnato la presenza cattolica in Italia, fin dalla unificazione del Paese, laddove il pensiero politico d'Oltralpe aveva fornito risposte ben diverse al radicale mutamento di clima seguito alla Rivoluzione francese.

1 A. Gramsci, I cattolici italiani, «Avanti!», edizione piemontese del 22 dicembre 1918, ora in Scritti politici, Editori riuniti, Roma 1978, vol. I, pp. 224-225; F. Chabod, L'Italia contemporanea (1918-1948), Einaudi, Torino 1961, p. 43. Per una analisi completa e approfondita delle valutazioni sulla nascita del Ppi si veda P. Bagnoli, Il PPI nel giudizio dei pensatori politici e degli storici contemporanei, «Rivista storica del socialismo», Nuova serie, 4 (1), $1^{\circ}$ maggio 2019, pp. 5-29.

Giuseppe Matulli, Italy, beppematulli38@gmail.com FUP Best Practice in Scholarly Publishing (DOI 10.36253/fup_best_practice)

Giuseppe Matulli, I cattolici e la politica fra le due guerre. Dalla lotta fra popolarismo e clerico-fascismo alla nascita della democrazia cristiana, pp. 1-22, (c) 2020 Author(s), CC BY 4.0 International, DOI 10.36253/97888-5518-202-7.03, in Piero Barucci, Piero Bini, Lucilla Conigliello (edited by), I mille volti del regime. Opposizione e consenso nella cultura giuridica, economica e politica italiana tra le due guerre, (c) 2020 Author(s), content CC BY 4.0 International, metadata CC0 1.0 Universal, published by Firenze University Press (www.fupress.com), ISSN 2704-5919 (online), ISBN 978-88-5518-202-7 (PDF), DOI 10.36253/97888-5518-202-7 
Risposte coerenti con il progressivo laicizzarsi della sfida politica, con l'articolarsi della società (fino ad allora suddivisa in corporazioni), in appartenenze culturali fra cui quella religiosa, e con l'avvio dell'età del costituzionalismo.

Secondo Paolo Pombeni ${ }^{2}$, le modalità del rapporto del mondo cattolico con la politica si rivelano in quattro ambiti distinti, se pure connessi. Due riguardano la Chiesa, e sono gli interventi in temporalibus e il «magistero ecclesiastico», cioè l'insegnamento della Chiesa in materia civile e sociale; gli altri due riguardano gli esponenti e i movimenti cattolici, ovvero il loro pensiero, e le azioni che ne seguono.

Il pensiero politico e civile dei movimenti cattolici si manifesta inizialmente soprattutto in Francia, in Belgio e in Germania. In particolare, in Francia nasce nel 1830 la rivista «Avenir» che, per la prima volta in ambito cattolico, sostiene la democrazia come valore cristiano (da cui la successiva indicazione del periodico come antesignano della $\ll$ Democrazia cristiana $\gg^{3}$ ); rivista, peraltro, già condannata nel 1832, insieme al suo fondatore Félicité Robert de Lamennais, da papa Gregorio XVI. L'iniziativa di Lamennais, cui aderiscono anche Charles de Montalembert e Henri Lacordaire, è espressione di quella parte del mondo cattolico che accetta il costituzionalismo postrivoluzionario, staccandosi dalla reazione ecclesiastica legittimista, sostenitrice di un ritorno alla società medievale, ma anche dalle tesi di Joseph De Maistre, che prefigura una società sottoposta alla «riconosciuta autorità del Papa». Le prime iniziative di esponenti e di movimenti cattolici si realizzano, soprattutto, in Francia e Germania.

In Italia il dato più significativo è la larga adesione popolare alla Chiesa cattolica ed è su questo cospicuo substrato che si innesta la lunga fase di dinamiche contrappositive, a colpi di azioni e reazioni, fra lo Stato italiano e il Papato, nonché fra il Papato e le iniziative del mondo cattolico in senso lato.

Alla formula cavouriana «libera Chiesa in libero Stato» risponde tre anni dopo, nel 1864, Pio IX con un atto del magistero: l'enciclica Quanta cura con annesso Sillabo, che condanna il liberalismo. Nel 1870, dopo la breccia di Porta $\mathrm{Pia}$, con un 'intervento in temporalibus', lo stesso pontefice vara il Non expedit (che avvia l'opposizione cattolica con la formula 'né eletti, né elettori'), dando vita di fatto a un movimento alle dipendenze della Curia romana, l'"Opera dei congressi", volto ad affrontare i problemi sociali del Paese in alternativa all'organizzazione statale. In realtà l'opposizione vaticana viene incardinata anche su un presupposto 'tecnico' (che sarà poi ripreso in Assemblea costituente, oltre 70 anni dopo, da Giuseppe Dossetti), relativo al diritto della Chiesa di affermare la propria natura di «ordinamento giuridico originario», necessario a garantirle libertà d'azione anche nelle relazioni internazionali, e di disporre di un territorio, per quanto ridotto, su cui esprimere la propria sovranità. Il periodo di attesa

2 P. Pombeni, in dialogo con M. Marchi, La politica dei cattolici dal Risorgimento a oggi, Città nuova, Roma 2015, pp. 41-42.

3 P.A. Graziani, Laicato cattolico e cultura politica. Una vicenda europea di due secoli, Portalupi editore, Casale Monferrato 2003, p. 28. 
di una soluzione alla «Questione romana» le consente così di protestare una 'continuità negata', nonché di proclamarsi 'prigioniera' di fronte agli altri Stati, e insomma di presentarsi come una sorta di governo in esilio.

Molto in sintesi, il pensiero politico e civile dei cattolici italiani si trova, in quella fase storica, articolato fra il cattolicesimo liberale - di cui Alessandro Manzoni, senatore del Regno e, dopo il '70, cittadino onorario di Roma, rappresenta il più illustre riferimento - el'area maggioritaria dell'«intransigentismo», quella dei difensori a oltranza della sovranità temporale della Santa Sede e della estensione del magistero papale a ogni ambito della via associata ${ }^{4}$.

La svolta arriva, nel 1878, con l'avvento al soglio pontifico di papa Leone XIII, che, prima con l'enciclica Immortale Dei (1885), poi con la più nota De rerum novarum (1891), pone le condizioni di una nuova stagione per quanto riguarda la presenza dei cattolici nella società, anche rispetto all'Opera dei congressi, inaugurando così la serie dei pontefici (con nomi diversi da «Pio», laddove «Pio» continueranno a denominarsi i pontefici 'accentratori', diffidenti dell'autonomia politica e sociale dei cattolici) rispettosi, o anche promotori, dell'autonoma iniziativa dei laici cattolici. Per opera di don Romolo Murri, e con l'intento esplicito di avviare la preparazione di un partito cattolico, nasce il movimento della «Democrazia cristiana», che nel 1905 darà poi vita alla «Lega democratica nazionale», cui aderiranno anche don Luigi Sturzo e Giuseppe Toniolo.

Nel 1903, però, a Leone XIII succede Pio X, che di nuovo impronta il suo pontificato a una chiusura totale ad ogni forma di autonomia organizzata, tanto da sciogliere anche l'Opera dei Congressi. In alternativa, come struttura totalmente dipendente dalla gerarchia ecclesiastica, Pio X lancia l'Azione cattolica, mentre ogni forma di modernizzazione del clero viene frontalmente avversata. Di lì a poco, Murri sarà prima sospeso a divinis (1907), poi scomunicato (1909), mentre la Lega democratica viene sconfessata.

È in questo clima che, nell'area del Bergamasco, si realizzano le prime intese per l'appoggio da parte degli elettori cattolici a candidati conservatori concordati, prima, limitata, eccezione al Non expedit. Sturzo reagisce e pronuncia nel 1905 lo storico discorso di Caltagirone, in cui sostiene la necessità che si ponga fine a ogni organizzazione clericale e che l'iniziativa dei cattolici si misuri attraverso la formazione di un partito, il quale - non ravvisandosi in Italia problemi di libertà religiosa - non si ponga a difesa degli interessi della Chiesa, bensì rappresenti «una tendenza popolare nazionale nello sviluppo del vivere civile», in competizione con tutti gli altri partiti. Un soggetto politico autonomo, insomma, che, pur riconoscendo il portato divisivo di una simile scelta all'interno del mondo cattolico, si schieri per la democrazia contro la conservazione.

$\mathrm{Nel}$ 1913, a un anno dalla riforma elettorale che ha introdotto il suffragio universale maschile, l'intesa per la selezione e l'appoggio cattolico a candidati

4 Celebre esempio di iconografia 'intransigentista' è l'immagine di don Davide Albertario arrestato e stretto fra due carabinieri, scelta da G. Spadolini come copertina del suo L'opposizione cattolica, Vallecchi, Firenze 1966. 
moderati trova una sua formalizzazione, valida in generale, nel Patto Gentiloni, grazie alla cui applicazione vengono eletti nove 'cattolici deputati' (come vengono appellati, allo scopo di non compromettere la gerarchia in un coinvolgimento troppo diretto nell'ambito politico). Fa parte di questo primo drappello Filippo Meda, che sarà ministro, prima delle Finanze, poi, dal 1916, del Tesoro, con i governi presieduti da Paolo Boselli e da Vittorio Emanuele Orlando, e che diventerà poi esponente del $\mathrm{Ppi}$.

\section{La Grande guerra e la nascita del Partito popolare}

Nel 1914 sale al soglio pontificio Benedetto XV, noto per il suo pacifismo definirà, nel 1917, la guerra, con efficace sintesi, «inutile strage» - che non aveva trovato seguito fra i cattolici i quali, come aveva annunciato l'Azione cattolica, avrebbero fatto 'il loro dovere'. Il nuovo Papa non intende porsi come punto di riferimento della presenza sociale e civile cattolica, e questo spiega perché assista senza ostilità - e dunque, di fatto, con sostanziale benevolenza, sebbene senza interventi diretti - alla nascita del Partito popolare, avallando così implicitamente la posizione di Sturzo nella violenta polemica con padre Agostino Gemelli e don Francesco Olgiati. I quali ultimi sostengono la necessità di una organizzazione politica di stampo sostanzialmente confessionale e clericale, direttamente collegata con la gerarchia attraverso un assistente ecclesiastico (come già avviene con le strutture dell'Azione cattolica), e accusano il futuro fondatore del Partito popolare, con la sua linea di separazione degli ambiti, di «mettere Cristo in soffitta».

Mentre i movimenti cattolici italiani, distaccandosi dal pacifismo di Benedetto XV, abbandonano il neutralismo originario (come, del resto, tutti gli altri movimenti, dai socialisti ai giolittiani), aderendo con entusiasmo al patto segreto con la Triplice intesa siglato a Londra dal Re e da Antonio Salandra, Alcide De Gasperi - allora membro del parlamento di Vienna e impegnato nella ricerca di una soluzione che scongiuri l'entrata in guerra - incontra sia il ministro degli esteri italiano, Sidney Sonnino, che lo stesso Benedetto XV. Con quest'ultimo concorda l'opportunità dell'appello papale per la tregua del Natale 1914, nella speranza di trasformarla in un armistizio duraturo.

In quanto rappresentante della minoranza nazionale italiana nel parlamento dello Stato multinazionale asburgico (una caratteristica che costituisce anche la premessa della sua futura battaglia europeista), il politico trentino ha ben chiara, per averla sperimentata, la distinzione fra «Nazione» e «Stato», e considera l'irredentismo, nella sua esaltazione dello Stato nazionale, come espressione di un nazionalismo generatore di guerra. Ciò non gli impedisce di lavorare per ottenere una soluzione pacifica, attraverso un referendum, per il passaggio del Trentino all'Italia, come già era accaduto per gli altri stati italiani preunitari ${ }^{5}$.

5 Per quest'atteggiamento il futuro statista subirà poi l'accusa da parte dei fascisti di essere un 'austriacante', accusa poi non disdegnata nemmeno dalla sinistra. 
Si è ricordato più sopra come l'imminente nascita del Partito popolare italiano, il 18 gennaio del 1919, sia stata annunciata da Antonio Gramsci nel lungo articolo intitolato I cattolici italiani pubblicato sull'«Avanti!» del 22 dicembre 1918, in cui il nuovo partito viene considerato un 'siluro' contro la classe dirigente liberale, con ciò decretandone il fallimento. La valutazione più immediatamente politica, che sostanzia il giudizio di Gramsci, è che la nascita del Ppi possa bloccare gli effetti del Patto Gentiloni, destinandolo perciò a restare un episodio storicamente definito, e impedire così la formazione di un blocco conservatore cattolico-liberale.

Di fatto, don Sturzo realizza nel 1919 quello che aveva proposto nel 1905, sintetizzando la sua proposta politica nell'Appello ai liberi e forti ${ }^{6}$, di cui vale la pena di ripercorrere in sintesi i punti fondamentali. Innanzi tutto l'incipit, quasi un preliminare, dedicato alla politica internazionale (con il sostegno alle posizioni del presidente americano Woodrow Wilson e la fiducia riposta nella Società delle Nazioni), seguito da una considerazione della guerra appena conclusa come evento che aveva completato l'unità del Paese, capace di consolidare la coscienza nazionale. Un secondo punto decisivo del documento è l'affermazione della «a-confessionalità» del nuovo partito che, oltre ad escludere, nel nome, ogni richiamo religioso, non prevede alcun collegamento con la gerarchia ecclesiastica, non si richiama ai cattolici, e addirittura sceglie i democratici contro i conservatori, pur nella piena consapevolezza che quella distinzione sarebbe stata divisiva del composito mondo cattolico. Ancora, il documento esprime un'apertura al mondo moderno, che prescinde completamente dal Sillabo e ritiene piena ed effettiva la libertà religiosa esistente in Italia, pur in presenza della ancora aperta Questione romana. Infine, esclude esplicitamente che la ragion d'essere del partito possa essere la difesa degli interessi della Chiesa. Da notare che il riferimento ai principi del cristianesimo, richiamato nella conclusione, è collegato all'idea della 'missione' di civiltà assegnata all'Italia nella battaglia contro gli imperialismi anarchici, il materialismo socialista e il liberalismo, vecchio e settario.

L'Appello ribadisce inoltre come la ragion d'essere del partito stia nella strategia che punta a sostituire le elitarie classi dirigenti liberali con le classi popolari; da cui il sostegno ad una rappresentanza elettorale proporzionale, con il voto alle donne, un Senato elettivo in rappresentanza di organismi nazionali accademici, amministrativi e sindacali, il riconoscimento giuridico delle classi, l'affermazione dell'autonomia comunale, la riforma delle provincie e il decentramento regionale. Alla proposta relativa alle autonomie territoriali e sociali, si accompagna quindi la previsione di una funzione centrale di coordinamento che assicuri l'unità nazionale, e dunque l'esclusione del federalismo.

In armonia con il cambio di classe dirigente, il Partito popolare viene insomma qualificandosi per una prospettiva riformista, che investe tutti gli ambiti della vita nazionale richiamati espressamente nel documento.

6 Partito popolare italiano, Appello ai liberi e forti, 18 gennaio 1919. 
Dopo aver sostenuto la riforma elettorale proporzionale, alle elezioni del 1919 il Ppi conquista 100 deputati (20,6\%), mentre il Psi, primo partito, raggiunge 156 seggi (32,3\%). Assieme, i due partiti coprono la maggioranza assoluta del parlamento, e senza l'appoggio di almeno uno dei due, nessun governo è in grado di avere la fiducia dell'assemblea.

In omaggio alle terre redente - sottoposte, dalla fine della guerra, all'amministrazione militare italiana, e ancora prive, quindi, di una compiuta organizzazione amministrativa che consenta alla popolazione residente di partecipare alle elezioni ${ }^{7}$ - nel giugno del 1921 viene chiamato a presiedere il primo congresso del Partito popolare Alcide De Gasperi.

Con l'obiettivo di stroncare anche a livello locale i residui del Patto Gentiloni, il Partito popolare - che nel 1920 aveva determinato la crisi del governo di Francesco Saverio Nitti, per poi partecipare con due ministri al successivo gabinetto Giolitti - conduce nello stesso anno, in occasione delle elezioni amministrative, una durissima battaglia contro i cattolici conservatori, sostenuti da numerosi vescovi veneti. Ė un successo, che molto deve al clima favorevole del pontificato di Benedetto XV, il quale, però, muore all'inizio del 1922, e al quale succede Pio XI che, invece, mostrerà insofferenza per l'autonomia civile e sociale dei cattolici.

Alle successive elezioni politiche, nel 1921, il Ppi vede aumentare i propri deputati a 107 (20,7\%), mentre i socialisti scendono a 124 (24,7\%), i comunisti conquistano 16 seggi, e il blocco nazionale promosso da Giolitti ne conquista 275 , di cui 35 fascisti e 10 nazionalisti.

Per le reciproche pregiudiziali fra i gruppi liberali, socialisti e popolari, il parlamento si rivela ingovernabile, e ci si avvia così alla crisi definitiva del regime parlamentare liberale. Contro il parere del Ppi, Filippo Meda, cui il Re ha proposto di formare un nuovo gabinetto, rifiuta l'incarico, aprendo la strada al secondo incarico a Luigi Facta. Durante la rapidissima crisi del governo che precede l'avvento di Mussolini, su Ordine nuovo del 27 luglio 1922 Palmiro Togliatti (che sigla P.T.) scrive: «[...] il tiranno bieco contro il quale dovranno insorgere tutte le energie che ancora vivono nelle moltitudini avrà un solo aspetto ed un triplice nome. Esso si chiamerà, insieme, Turati, don Sturzo e Mussolini» ${ }^{8}$.

\section{L'avvento del fascismo: la lotta del Partito popolare col clerico-fascismo}

Falliti i tentativi, nel giugno del 1922, di formare governi di coalizione con i socialisti, all'inizio di ottobre Sturzo rifiuta di partecipare al governo Giolitti di salute pubblica (comprendente anche il Pnf), e il 28 ottobre arriva, con la marcia su Roma, l'incarico a Benito Mussolini.

Di fronte all'avanzata dei fascisti, emerge immediatamente la divergenza fra De Gasperi e Sturzo. Il primo, infatti, fin dal 1921 era convinto della possibili-

De Gasperi, infatti, potrà presentarsi alle elezioni soltanto nel 1921.

G. De Rosa, Il Partito popolare italiano, Laterza, Bari 1972, p. 130, nota 8. 
tà di «costituzionalizzare» e dunque riassorbire il fascismo (anche in ragione della sua contenuta dimensione di massa) nell'alveo istituzionale, in relazione al venir meno, con lo sfiammarsi del 'biennio rosso', anche dei pretesti dell'estremismo nero. Nell'immediato, insomma, il politico trentino non vede alternative all'avvento del fascismo, se non un governo di salute pubblica che sia in grado prima di contenerlo, e poi di annullarlo. Sturzo, invece, non la pensa così. Considera inutilizzabile la classe dirigente liberale per quella finalità, e si preoccupa soprattutto di ribadire la validità di una strategia popolare alternativa, appunto, sia al fascismo che alla classe dirigente che se ne era lasciata travolgere.

Per otto mesi la divergenza fra i due maggiori esponenti popolari si esprime nel formale rispetto dei diversi ruoli: Sturzo, segretario nazionale, tiene il Ppi in una posizione pregiudiziale nei confronti del governo Mussolini, pur rispettando i deputati che partecipano a titolo personale a quel governo; De Gasperi, presidente del gruppo parlamentare, è invece consenziente, e teso ad ogni possibile compromesso per salvare la legittimità costituzionale dello Stato.

Nel frattempo, fra i tanti che salutano positivamente l'ordine' portato dalla marcia su Roma c'è, in prima fila, la gerarchia ecclesiastica, che instaura subito un rapporto molto positivo col fascismo, e non solo in vista di una soluzione della annosa Questione romana. Intorno a un Mussolini presentato come «uomo inviato dalla Provvidenza», subito prende forma una chiara linea clerico-fascista, mentre il nuovo Presidente del Consiglio, valutando che la ragion d'essere del Ppi sia proprio la difesa degli interessi della Chiesa, è convinto che una generosa accoglienza delle richieste della gerarchia possa costringere don Sturzo, a meno di non far scomparire il Partito popolare, ad appoggiare il governo fascista.

Sturzo procede col partito a un'offensiva molto lucida. A meno di due mesi dalla formazione del governo Mussolini, il 20 dicembre del '22, in un discorso a Torino precisa la posizione politica del $\mathrm{Ppi}$ sottolineando la gravità dell'attacco al parlamento sferrato dal futuro Duce («un'aula sorda e grigia»), e affermando come con ciò il nuovo capo del governo abbia «ferito una rappresentanza» colpendo però «gli effetti, non la causa» di una situazione ormai al limite della legittimità costituzionale, portata fino a quel punto dalla crisi dello Stato liberale. In difesa della quale legittimità, quindi, la partecipazione al governo dei deputati popolari e degli altri gruppi liberali sarebbe risultata del tutto insufficiente.

Il braccio di ferro con i clerico-fascisti si manifesta anche all'interno del gruppo parlamentare. Le manovre di resistenza del Ppi all'assorbimento da parte fascista devono evitare la rottura del gruppo, che rischierebbe di agevolare una iniziativa ostile - come da richiesta di Mussolini - da parte della gerarchia ecclesiastica. Sturzo riesce a superare le opposizioni interne e celebra il congresso del Ppi che si apre il 12 aprile 1923 rivendicando la qualità culturale e politica della strategia popolare, anche esaltando la legge elettorale proporzionale come suo punto qualificante, e suscitando, come era inevitabile, la negativa reazione di Mussolini. Il quale definisce il discorso di Sturzo come quello di un «nemico» e pone fine alla collaborazione di governo con i ministri popolari.

Il congresso di Torino del Ppi rappresenta (come subito riconoscono esponenti liberali e socialisti quali Giovanni Amendola, Filippo Turati, oltre a Mario 
Missiroli) la prima, aperta, denuncia politica del carattere reazionario e totalitario del fascismo, del resto apertamente rivendicato dallo stesso Mussolini, che considera perciò quella denuncia il riconoscimento di fatto del fascismo come movimento «antiparlamentare, antidemocratico, antiliberale» ${ }^{9}$ da lui stesso apertamente proclamato tale.

All'adesione al Ppi e a Sturzo da parte delle forze politiche non fasciste, si contrappone una totale chiusura al progetto politico popolare da parte della gerarchia ecclesiastica. La esplicita richiesta fascista dell'allontanamento di Sturzo è avallata dalla stampa clerico-fascista, tanto che lo stesso De Gasperi, in una intervista al «Corriere della sera» del 27 giugno del 1923, mentre conviene nel considerare discutibile che un sacerdote sia segretario di un partito politico, osserva come, in quella fase, un eventuale ritiro di Sturzo non deciso autonomamente dal Ppi avrebbe prodotto due inevitabili (e inaccettabili) significati: l'autocertificazione della 'minorità politica' dei cattolici italiani e il diritto di fatto della Santa Sede di interferire negli affari italiani «di modo che», osserva De Gasperi, «qualsiasi governo si sentirebbe autorizzato a farsi valere attraverso gli interessi religiosi». Il 5 luglio, però, con una lettera del cardinal Gasparri al gesuita Pietro Tacchi Venturi, Pio XI fa sapere a Sturzo che sarebbe gradito un suo disimpegno «senza ulteriore dilazione» dalla segreteria del partito; a nulla vale la dignitosa risposta che Sturzo invia direttamente a Pio XI, avendo premesso il suo atto di obbedienza ${ }^{10}$. Il 10 luglio 1923 il sacerdote si dimette da segretario (sostituito da un triunvirato composto da Giovanni Gronchi, Giulio Rodinò e Giuseppe Spataro), e il giorno successivo l'«Osservatore romano» valuta quelle dimissioni come un contributo alla pacificazione degli animi.

Nel frattempo, fra minacce e violenze si apre l'ultima prova di forza parlamentare: la discussione sulla legge maggioritaria, nota come 'legge Acerbo', che punta ad assicurare la maggioranza assoluta a chi conquisti il $25 \%$ dei voti validi, e che porterà a una clamorosa scissione del gruppo parlamentare popolare, una parte del quale, dopo aver ottenuto l'impegno del gruppo ad astenersi proprio

9 G. De Rosa, L'avvento del fascismo, Laterza, Roma-Bari 1987 (vol. III di A. De Gasperi, C. De Mita, G. De Rosa, I cattolici dall'opera dei congressi alla Democrazia cristiana. Dal 1870 al 1986) a p. 85, alla nota 23, riporta quanto scrisse Mussolini in Tempi della rivoluzione fascista, Alpes, Milano 1930: «il carattere antiparlamentare, antidemocratico, antiliberale» del fascismo stesso assunse «immediato rilievo» nel congresso di Torino «alle prime incompatibilità» dei popolari con il fascismo. Alla successiva p. 87 De Rosa cita la lettera di Giovanni Amendola a Turati del 15 aprile 1923: «Il congresso di Torino ha già compiuto la funzione di protesta per la libertà. È stata la prima ondata, prima di lanciare la seconda è bene vedere in qual modo reagirà il duce» (tratta da Filippo Turati attraverso le lettere dei corrispondenti. 1880-1925, a cura di A. Schiavi, Laterza, Bari 1947, pp. 232-233). Alla stessa pagina, nella nota 1, De Rosa riporta l'opinione di Mario Missiroli secondo cui il Ppi si era rivelato «il solo partito democratico organizzato», e Sturzo si era assunto «il compito ben difficile di difendere le giuste e moderate tesi fondamentali della democrazia del paese» (tratta da M. Missiroli, Polemica liberale, Zanichelli, Bologna 1954, p. 313).

10 L. Sturzo, Lettere non spedite, a cura di G. De Rosa, il Mulino, Bologna 1996, pp. 267-275. 
per evitare rotture interne, rompe il patto e vota a favore della legge, consentendone così l'approvazione.

Ad onta del progetto mussoliniano di assegnare in esclusiva al proprio governo la tutela degli interessi della Chiesa, col risultato di vanificare l'istanza politica del Partito popolare, la struttura periferica del Partito popolare regge. Il che stimola da un lato un crescendo delle violenze fasciste contro le sezioni e i militanti popolari, e dall'altra un pressing sempre più ravvicinato sul Vaticano perché appoggi l'area clerico-fascista.

Le elezioni del 1924 segneranno il successo del 'listone' guidato dal Pnf (con il 64\%), mentre il Ppi, pur dimezzando il proprio peso parlamentare rispetto al 1921 (9,6\%), si conferma come il più consistente dei partiti di opposizione; il Psi si è infatti diviso, mentre il Partito comunista è al 3,7\% ${ }^{11}$.

Il 27 giugno del 1924, a seguito del delitto Matteotti, prese corpo la secessione Aventiniana ove inizia il tentativo di collaborazione fra socialisti e popolari, e nel frattempo si sviluppa una violenta polemica fra «Il Popolo» e la «Civiltà cattolica» contro le aperture dei popolari verso i socialisti. Protagonista delle trattative per l'intesa Psi-Ppi, De Gasperi entra nel mirino della Santa Sede, attaccato in un discorso agli studenti universitari (riportato su l' $\ll$ Osservatore romano» il 9 e ribadito il 17 settembre) dallo stesso pontefice Pio XI, intervenuto anche per determinare l'esilio 'volontario' di Sturzo. Il sacerdote, ex segretario del Ppi, sarà costretto a lasciare l'Italia il 25 ottobre 1924 (rientrerà nel suo paese soltanto nel 1946). Di fronte al successo della strategia contro i popolari, il Ppi è privato anche del direttore de «Il Popolo», Giuseppe Donati, il quale, per mettersi in salvo dopo la sua denuncia delle responsabilità fasciste nel delitto Matteotti, fugge dall'Italia.

Nel clima seguito al discorso di Mussolini del 3 gennaio 1925, che segna di fatto la fine della legalità liberale e l'inizio del regime, l'11 giugno De Gasperi, Colonna di Cesarò e Giovanni Amendola vanno in udienza da Vittorio Emanuele III a denunciare inutilmente le violazioni fasciste dello Statuto Albertino.

In tale clima, fra il 28 e il 30 giugno, De Gasperi indice il quinto congresso del Partito popolare (che sarà anche l'ultimo). Un congresso, si può dire, 'a futura memoria', il cui solo fine è di ribadire la resistenza alla deriva clerico-fascista che, forte dell'antica scomunica del Sillabo, tende a considerare il fascismo come la risposta più idonea all'«errore liberale».

Pur esprimendo rispetto - per evidenti ragioni tattiche - per la scelta apolitica dell'Azione cattolica, De Gasperi non rinuncia a difendere l'autonomia del Ppi e la linea fin lì perseguita dal partito. E ribadisce la sua interpretazione del momento storico:

11 Dal 1919 al 1924 il Ppi perde 11,1 punti percentuali scendendo dal 20,7\% del 1921 al 9,6\%; il Psi (che ha espulso Turati il quale fonda il Psu e raccoglie subito il 5,9\%) passa dal 32,3\% del 1919 al 5,0\%, mentre il Pc raccoglie il 3,7\%; la battaglia clerico-fascista sembra dunque incidere meno sul consenso elettorale del Ppi, di quanto il presunto laburismo di Mussolini riesca a incidere sull'elettorato socialista, particolarmente nella sua componente sindacale. 
il contrasto non è fra fascismo e liberalismo, come scuola e metodo transeunte, ma tra il fascismo e alcune esigenze fondamentali dell'organizzazione politica moderna. È insomma, il contrasto fra lo Stato di diritto quale si è sviluppato nelle costituzioni moderne e il vecchio Stato di polizia, che tenta ora di ricomparire sotto mutate spoglie.

Ricorda, quindi, il contributo assicurato dalle formazioni cattoliche alle moderne costituzioni, a Weimar come a Vienna, a Praga come a Belgrado e a Varsavia, e, sottolineando come il Ppi non faccia eccezione, ribadisce che «non è quindi un duello fra il liberalismo e il nazional fascismo, al quale noi dovremmo assistere come spettatori neutrali e disinteressati $\gg^{12}$; la prospettiva è dunque: $\ll[\ldots]$ tenere fermo fino alla fine».

Al congresso assiste Piero Gobetti, che esalterà il Ppi e la figura di De Gasperi su $\ll$ La rivoluzione liberale $\gg^{13}$.

\section{De Gasperi antifascista: dal carcere alla Biblioteca Vaticana, inizia la 'lunga vigilia'}

Dopo il congresso del giugno, le minacce e le violenze impongono a De Gasperi di lasciare sia la direzione de «Il nuovo Trentino» che la segreteria del Ppi, anticipando, di poco, le cosiddette 'leggi fascistissime' con cui, fra il 24 dicembre 1925 e il novembre 1926, si formalizza la dittatura. Inizia così la cosiddetta 'lunga vigilia' del politico cattolico.

Degli esponenti del Partito popolare, dopo Sturzo e Donati anche Francesco Luigi Ferrari prenderà, nel '26, la via dell'esilio (Donati morirà in Francia nel 1931, Ferrari in Belgio nel 1933), mentre gli altri esponenti del Ppi sono costretti all'attività privata; se per professionisti come Filippo Meda, Adone Zoli, Attilio Piccioni, Giuseppe Spataro, che sono avvocati, la vita sarà meno dura, per altri, come Giovanni Gronchi, si tratterà di rinunciare all'insegnamento e inventarsi un'attività commerciale.

Quanto a De Gasperi, si ritira in Valsugana; ma dopo l'attentato di Bologna a Mussolini del 31 ottobre 1926 (attribuito, come è noto, ad Anteo Zamboni), sarà vittima di una spedizione punitiva che, nella notte fra il 5 e il 6 novembre, lo porterà, assieme al fratello Augusto, a ritrovarsi di fronte al federale di Vicenza, accusato di opporsi al governo Mussolini e sottoposto ad una sorta di processo. Solo l'intervento del deputato fascista Paolo Marzotto, più moderato degli altri, gli eviterà il peggio. Marzotto preleva i due fratelli e li fa accompagnare alla stazione ferroviaria.

12 G. Vecchio, Alcide De Gasperi,1918-1942. Le sconfitte di un politico di professione, in A. De Gasperi, Scritti e discorsi politici. Edizione critica, vol. II. Alcide De Gasperi dal Partito popolare italiano all'esilio interno, 1919-1942, a cura di M. Bigaran e M. Cau, il Mulino, Bologna 2007, p. 132 (citato in A. De Gasperi, Diario 1930-1943, a cura di M. Sergio, il Mulino, Bologna 2018, p. 26).

13 «La rivoluzione liberale», n. 27, 5 luglio 1925, ora in Scritti politici, a cura di P. Spriano, Einaudi, Torino 1969, pp. 859-863. 
Ma la situazione precipita presto. Dopo il diniego (dichiarato con nota a mano dello stesso Mussolini) al rilascio del passaporto, con cui sperava di raggiungere le terme di Vichy, De Gasperi, insieme alla moglie, è arrestato alla stazione di Firenze l'11 marzo 1927, e incarcerato con l'accusa di tentativo di espatrio. La sentenza lo condanna a quattro anni, ridotti in appello a due e mezzo, di cui sconterà solo sedici mesi grazie all'intervento dell'amico monsignore Celestino Endrici, vescovo di Trento, il quale chiederà pubblicamente al Re, durante la cerimonia per l'inaugurazione del monumento a Cesare Battisti, un intervento in favore del politico trentino. De Gasperi è scarcerato nel luglio del 1928, ma rimarrà 'sorvegliato speciale' fino al 1933, con divieto di allontanarsi da Roma, e afflitto da notevoli problemi economici.

Pochi mesi dopo la sua scarcerazione, la firma dei Patti lateranensi gli suscita commenti molto netti. Il giorno successivo, il 12 febbraio del 1929, De Gasperi scrive all'amico trentino don Simone Weber:

[...] i cocchi dei trionfatori passano schizzando fango sui travolti che stentano a salvarsi agli angoli della via [...] I cattolici di qui sono variamente commossi, $i$ vecchi popolari sono furibondi, [...] i temporalisti più accesi, compresi i Gesuiti, portano intorno una faccia trionfale [...] Quindi contenti i fascisti, contenti i massoni, Mussolini è trionfante $[\ldots]^{14}$.

E tuttavia, egli riesce a collocare la firma del Concordato (compresi i suoi aspetti più negativi, in particolare il trattato, e le sue concessioni finanziarie) in una prospettiva storica, riconoscendo l'innegabile successo ottenuto dal regime con l'accordo, e osservando d'altra parte come Mussolini abbia potuto approfittare della positiva evoluzione dei rapporti fra Stato e Chiesa, in corso già da tempo e che avrebbe comunque portato a una soluzione. Il vantaggio ottenuto dal leader fascista, insomma, pure dannoso per il Paese, non impedisce di giudicare il concordato come storicamente inevitabile, al punto che lo stesso Sturzo, a giudizio di De Gasperi, avrebbe firmato il Patto se fosse stato al posto del Papa. In altri termini: il dente era tolto, e la politica italiana ne avrebbe tratto profitto.

Il 3 aprile del '29, a Concordato varato, De Gasperi è assunto alla Biblioteca Vaticana con un incarico inizialmente molto modesto, come la redazione di cataloghi, per il quale riceve un compenso molto basso (è infatti costretto a integrarlo con traduzioni di libri dal tedesco) e con la 'necessaria' prudenza di non lavorare nelle sale con accesso al pubblico. Da notare che questa collocazione rappresenta la sola modalità con cui, essendo posto sotto sorveglianza speciale per aver tentato di espatriare clandestinamente, De Gasperi era riuscito a conquistarsi una 'extraterritorialità'. Ne sarà infatti grato a Pio XI, che aveva respinto le proteste del governo per quell'assunzione. La sua presenza nella biblioteca, tuttavia, rimase un costante argomento di polemica da parte del regime, che aveva preteso l'emarginazione da ogni incarico nelle organizzazioni cattoliche di tutti gli esponenti del disciolto Partito popolare e, nonostante le rassicurazioni

14 A. De Gasperi, Lettere sul Concordato, Morcelliana, Brescia 1970, pp. 59-61. 
del Vaticano, temeva l'influenza politica che l'ex segretario del Ppi avrebbe potuto esercitare stando a Roma ${ }^{15}$.

L'impegno nella Biblioteca Vaticana, pur nelle condizioni ricordate, consente a De Gasperi un punto di osservazione privilegiato sullo scenario travagliato degli anni Trenta, dalla Germania di Weimar con l'avvento del nazionalsocialismo, alla tragedia austriaca di Engelbert Dollfuss, a tutte le altre vicende di quell'epoca tormentata che diventeranno oggetto della rubrica quindicinale che il 'bibliotecario' curerà, con lo pseudonimo di Spectator ${ }^{16}$, sulla «Illustrazione vaticana». Quegli anni gli consentirono anche di proseguire ricerche storiche e riflessioni (che appariranno, in varie riviste, con pseudonimi diversi) su argomenti cruciali per la vita culturale, civile e politica del Paese: dalla valutazione critica della Storia d'Europa di Benedetto Croce, alle sorti del protestantesimo nel terzo Reich e dei movimenti sociali cristiani nei diversi contesti nazionali (Francia, Belgio, Austria, Germania), alla storia degli ultimi papi, alle sorti del Partito popolare, alla crisi della fragile democrazia prefascista ${ }^{17}$.

Una speciale attenzione De Gasperi dedica allo studio del 'Centro' tedesco (Zentrum), di cui ricostruisce la storia: dall'opposizione all'imperialismo del 'cancelliere di ferro' Otto von Bismarck, in difesa dello Stato di diritto e del nuovo corso del costituzionalismo, fino al sacrificio imposto al Centro dagli accordi diretti fra Bismarck e Leone XIII ${ }^{18}$ e all'impegno del Centro nella collaborazione con i socialisti nella costituente di Weimar. L'analisi critica porta continuamente il politico, calato anche nei panni dello storico, a rilevare le sempre incombenti tentazioni clericali e confessionali presenti sulla scena politica tedesca, che alla prova delle esperienze concrete il movimento cattolico sarà tuttavia capace di

15 De Gasperi, Diario 1930-1943, cit., pp. 29-33.

16 G. Tupini, De Gasperi. Una testimonianza, il Mulino, Bologna 1992, p. 43.

17 In A. De Gasperi et al., I cattolici dall'opera dei congressi alla Democrazia cristiana. Dal 1870 al 1986, vol. IV. I cattolici dall'opposizione al governo, Laterza, Roma-Bari 1987, pp. XII-XIII sono contenute le indicazioni bibliografiche originali di alcuni degli scritti di De Gasperi nella 'lunga vigilia', riportati nel volume stesso: Ripensando la "Storia d'Europa", pubblicato in «Studium», maggio-giugno 1932, pp. 248 e seguenti, con lo pseudonimo V. Bianchi; I tempi e gli uomini che prepararono la Rerum Novarum, edizioni di Vita e pensiero, 1928, poi 1931, sotto lo pseudonimo di M. Zanatta; Un maestro del corporativismo cristiano, «Rivista internazionale di scienze sociali e discipline ausiliarie», gennaio 1928, con lo pseudonimo G. Jaspar; Il Centro germanico, «Rivista internazionale di scienze sociali e discipline ausiliarie», 8 agosto 1928, sempre a firma G. Jaspar; Cronache internazionali, sotto il titolo «Quindicina internazionale», tra il gennaio 1933 e l'ottobre del 1938, nel periodico «Illustrazione vaticana» a firma Spectator; Siate voi stessi! Siate ottimisti!, appello ai giovani pubblicato nel 1934 su un numero unico di «Associazione universitaria», a firma G. Fortis. Poi, nel periodo clandestino, gennaio-febbraio 1944, escono su «Il Popolo» tre articoli a firma Demofilo, raccolti sotto il titolo Rinascita della Democrazia cristiana.

18 De Gasperi sottolinea come il leader del Zentrum, Ludwig Windthorst, difendendo l'autonomia delle sue valutazioni politiche rispetto alle pressioni del pontefice, chiedesse che ove la scelta dell'autonomia (come egli non credeva probabile) avesse dovuto pregiudicare le sorti del partito, sulla tomba del Centro avrebbe dovuto essere scritto «dai nemici mai vinto, ma dagli amici abbandonato». 
superare $^{19}$. Lo stesso De Gasperi fornirà, a libertà riconquistata, la chiave d'interpretazione dei suoi scritti di quel periodo:

Lo studio sul 'Centro' germanico e quelli sul corporativismo sono comparsi in pieno periodo fascista e sotto diversi pseudonimi, anzi i primi due furono scritti in una clinica mentre ero guardato a vista dai carabinieri (1928). I lettori devono considerare bene le date. Vi si vede ancora lo sforzo di evitare qualsiasi accenno che potesse compromettere la pubblicazione o aggravare ulteriormente la sorte dell'autore condannato pochi mesi prima a quattro anni di prigione. Nella sintesi storica sull'organizzazione politica del 'Centro' tedesco è chiara la tendenza di ricordare ai cattolici l'esempio delle lotte parlamentari sostenute da Windthorst e compagni contro la formidabile dittatura di Bismarck, e negli studi sul corporativismo (1933-34) l'autore ha cura di dimostrare - senza avere l'aria di farlo - che quanto vi fosse di buono nella concezione corporativa risaliva a più di mezzo secolo addietro, e che in ogni caso lo spirito del sistema fascista asservito alla dittatura del partito unico era essenzialmente diverso; cose che si potevano insinuare solo con molta cautela, ma che spero abbiano contribuito a mettere in guardia qualche cattolico troppo ingenuo o troppo corrivo a transigere col fascismo per certe somiglianze di organizzazione formale $[\ldots]^{20}$.

Tutte le vicende che caratterizzano i continui compromessi col fascismo da parte del Vaticano e delle organizzazioni cattoliche sono registrate da De Gasperi in un sintetico diario pubblicato soltanto recentemente (2018), dal quale si desume fra l'altro il suo soffertissimo giudizio sull'accordo con cui il regime, oltre a gratificare la Chiesa con una aperta benevolenza per la scuola privata, aveva ottenuto l'iscrizione automatica alle organizzazioni giovanili fasciste (come i 'balilla') di tutti gli alunni iscritti alle scuole cattoliche. Il che, sul versante privato, lo portò - con grande disagio personale e dell'intera sua famiglia - ad iscrivere le figlie all'unico istituto religioso, a quanto risulta, riuscito a sottrarsi a quell'automatismo, ovvero le Suore di Never.

\section{La preparazione del 'dopo'}

La politica attiva di De Gasperi era terminata col 'congresso a futura memoria' del giugno del '25, ma la 'lunga vigilia' rappresenta una sorta di periodo di incubazione di quelle che diverranno in seguito evidenti, in lui, le qualità tipiche ed essenziali del politico, secondo la definizione datane da Max Weber; e cioè «la lungimiranza», «il senso di responsabilità»e e «la passione», da collocare nel quadro valoriale di un'etica della responsabilità inscindibile dall'attività politica stessa.

La passione emerge in modo assai chiaro, in particolare, in una lettera dal carcere indirizzata alla moglie, in cui De Gasperi definisce la sua 'carriera', o

19 A. De Gasperi, Il centro germanico, in Id. et al., I cattolici dall'opposizione al governo, cit., pp. 251-331.

20 A. De Gasperi, Studi e appelli della lunga vigilia, Magi-Spinetti, Roma 1946, pp. 5-6. 
meglio la sua 'missione', quali che fossero le condizioni in cui operare ${ }^{21}$, il totalizzante impegno politico che costringe la famiglia nelle ristrettezze, delle cui conseguenze è convinto, perciò, di non doversi incolpare.

Quanto alla virtù weberiana della lungimiranza, essa emergerà con chiarezza alla caduta del fascismo, con la fine della stagione clerico-fascista che mette però in evidenza come il mondo cattolico fosse, in generale, ancora irretito in un consolidato atteggiamento antiliberale, retaggio, almeno in parte, della storica condanna del liberalismo da parte delle gerarchie. Proprio la capacità di guardare oltre la superficie dei fenomeni consente a De Gasperi di collocare e interpretare le tendenze più conservatrici all'interno di un più vasto contesto di orientamenti diversi, presenti nel mondo cattolico fin dagli anni '30. La corrente più immune dalle tendenze antiliberali era quella dei «popolari» che - come si è ricordato - si poneva in alternativa alla classe dirigente liberale, con l'obiettivo di sostituirla nel governo democratico di una società pluralista, operante in ambito economico attraverso il mercato. Un'altra corrente era quella che si era formata nell'alveo della Federazione degli universitari cattolici italiani (Fuci), il cui assistente, monsignor Giovanni Battista Montini, aveva discusso con i giovani universitari (molti dei quali, a cominciare da Aldo Moro, andranno a costituire la classe dirigente dell'Italia repubblicana) i testi di Jacques Maritain, e in particolare, nel 1936, Umanesimo integrale, l'opera più nota del filosofo francese. La tesi di Maritain, che, come è noto, nella interpretazione del messaggio evangelico distingue le indicazioni religiose proprie del magistero della Chiesa dalle valutazioni sociopolitiche che attengono alla responsabilità autonoma dei singoli fedeli, risulta evidentemente troppo 'liberale' per i paradigmi delle gerarchie cattoliche. $E$ determinerà infatti contro il filosofo (ed ex ambasciatore di Francia presso la Santa Sede) un successivo intervento vaticano, con il divieto all'Università Cattolica di assegnargli una laurea honoris causa, e l'avvio delle procedure di scomunica, poi interrotte dalla scomparsa di Pio XII ${ }^{22}$.

Nel solco della tradizione del Partito popolare, De Gasperi è insensibile alla ricerca di soluzioni che individuino esclusivamente nel liberalismo e nel comunismo sovietico i termini di riferimento negativi dell'azione politica dei cattolici, riuscendo con ciò ad evitare antistorici e velleitari richiami al corporativismo. Fondandosi sul principio dell'autonomia della dimensione politica, i popolari, nella sua visione, sono chiamati non già a restringere la ricerca ad una ipotetica 'terza via', ma a tradurre i valori civili e sociali derivati dal messaggio evangelico in proposte politiche 'aperte', capaci di dialogare con le altre culture. E perciò stesso di porre le basi (come lo stesso De Gasperi aveva messo in rilievo nell'analisi dei movimenti cattolici in Europa) per la realizzazione anche in Italia di un nuovo regime costituzionale, innegabilmente fondato su valori maturati nell'alveo della cultura liberale, a sua volta incubata dalla Rivoluzione francese.

\footnotetext{
21 A. De Gasperi, Lettere dalla prigione. 1927-1928, Cinque Lune, Roma 1974, p. 101.

22 F. De Giorgi, Paolo VI. Il papa del moderno, Morcelliana, Brescia 2015, pp. 233-234.
} 
La lungimiranza degasperiana si spinge così a leggere perfino nel dirompente evento che inaugura l'età contemporanea un'attuazione dei valori evangelici ${ }^{23}$.

Un'apertura niente affatto consueta in ambito cattolico, soprattutto italiano, che consentirà al futuro leader democristiano di definire se stesso «un cattolico ortodosso e credente [che] attraverso l'illuminazione dell'esperienza altrui e della propria divenne politicamente umanista e ricettivo di ogni cosa buona e di ogni fede nella libertà e nella tolleranza civile $\gg^{24}$. E di cui De Gasperi dà prova - tanto più significativa dati l'affetto e la stima che lo legavano a Benedetto Croce $^{25}$ - anche in una lettera a Stefano Jacini del 27 marzo del 1932 nella quale, riprendendo la critica alla Storia d'Europa ${ }^{26}$, contesta al filosofo napoletano di aver letto le vicende del mondo cattolico soltanto sulla base degli atteggiamenti della gerarchia ecclesiastica:

Leggo il Croce e mi arrabbio [...] perché questo suo libro antipretino è il libro più clericale che sia mai stato scritto [...] a sentire il Croce - ed è un maestro tra i molti - nessun credente nella vita ventura può essere un liberale cosciente, sai dirmi - chiede all'amico - ove potremmo collocarci noi ed un discreto numero di nostri antenati spirituali $?^{27}$

Ritornando nel 1932 sull'argomento col saggio Ripensando la 'storia d'Eu$\mathrm{ropa}^{28}$, in cui ricorda la presenza di correnti liberali nella storia del movimento cattolico (Lacordaire, Montalembert), De Gasperi contrappone a Croce Le modern democracies di James Bryces, studioso inglese 'a-cattolico' che accusa Voltaire e Rousseau di non aver colto il valore della fede religiosa come contributo alla consapevolezza della dignità e della libertà della persona umana. $\mathrm{E}$ in occasione della ripubblicazione, nel 1946, della recensione all'opera di Croce, scriverà: «Formalmente l'articolo è una critica contro certe affermazioni di Croce,

$23 \ll[\ldots]$ ed ecco che nella Rivoluzione si scopre il fermento evangelico della giustizia e della verità: libertà personale, autogoverno della Nazione, libero suffragio, divisione e indipendenza dei poteri, pace operosa e non guerre [... ]», A. De Gasperi, I presupposti storici e ideali della Dc, discorso conclusivo del Consiglio nazionale Dc di Fiuggi dell' 1 agosto 1949, in Scritti e discorsi politici. Edizione critica, vol. IV. Alcide De Gasperi e la stabilizzazione della Repubblica, 1948-1954, a cura di S. Lorenzini e B. Taverni, il Mulino, Bologna 2009, tomo I, pp.1264-1271.

24 A. De Gasperi, De Gasperi scrive. Corrispondenza con capi di stato, cardinali, uomini politici, giornalisti, diplomatici, a cura di M.R. De Gasperi, Morcelliana, Brescia 1974, vol. I, p. 28, lettera di De Gasperi a Mario Vinciguerra del 22 novembre 1950.

25 F. Mazzei, Amicizie e corrispondenze liberali di De Gasperi alla Biblioteca Vaticana, in P. Ballini (a cura di), Quaderni degasperiani per la storia dell'Italia contemporanea, Rubettino, vol. VI, Saveria Mannelli 2017, pp. 113-117.

26 B. Croce, Storia di Europa nel secolo decimonono, Laterza, Bari 1932.

27 Lettera a Stefano Jacini riportata in G. Vecchio, Esule in patria: gli anni del fascismo (19261943), in A. Canavero et al., Alcide De Gasperi, Rubettino, Saveria Mannelli 2009, vol. I, pp. 580-588.

28 A. De Gasperi, Ripensando la storia d'Europa, originariamente pubblicato sulle riviste «Hochland» e «Studium», 33 (5-6), 1932, pp. 248 e sgg. con lo pseudonimo V. Bianchi, ora in De Gasperi et al., I cattolici dall'opposizione al governo, cit., pp. 557-574. 
ma è chiaro che sotto questa veste polemica l'autore mira a ricordare ai cattolici gli uomini più insigni della loro scuola che nel secolo XIX parlarono e agirono per la libertà politica e la dignità umana $\gg^{29}$.

Il profondo senso di appartenenza alla Chiesa riguarda, in De Gasperi, la più ampia comunità ecclesiale, in cui anche i riformatori trovano spazio, sebbene spesso (e però non sempre) in contrasto con la gerarchia. La cui struttura politico-diplomatica, d'altra parte, pur rilevante dal punto di vista dell'analisi politica, lo statista cattolico valuta del tutto secondaria rispetto alla missione spirituale e sociale della Chiesa nel mondo. E per quanto lo riguarda, una cosa è certa: appunto in quanto gli è possibile riconoscersi fino in fondo nella missione spirituale della Chiesa, gli è anche possibile - spiega - ritenersi libero e autonomo nel valutare con realismo il ruolo della Chiesa stessa nello scacchiere politico nazionale.

È sulla base di questi presupposti che, in un incontro del 1938, De Gasperi rivela lo schema di una strategia politica che getta le basi delle sue azioni future. Lo testimonia Adriano Ossicini ${ }^{30}$, che riferisce così le parole dell'ex segretario del Ppi:

Il fascismo cadrà per crisi interna, per le drammatiche posizioni che sta prendendo. Però noi cattolici dobbiamo stare molto attenti, perché abbiamo fallito al momento del secondo governo Facta quando Meda fu officiato per prendere il potere. Rifiutammo di andare al governo. Avremmo potuto tentare, non so se non avevamo la forza sufficiente per farlo.

De Gasperi, secondo Ossicini, dice di non condividere l'analisi del fascismo compiuta dal Pci, secondo cui esso non sarebbe che uno strumento al servizio della borghesia italiana. Ricorda il futuro esponente del movimento cattolico comunista: «Egli pensava che il fascismo si fosse affermato nel 1922 perché non si era offerta alla borghesia una differente e più democratica prospettiva». E che alla borghesia, dunque (la cui solida egemonia non lasciava intravvedere, al momento, alcuna possibile alternativa), si sarebbe dovuta mettere a disposizione, una volta caduto il regime, una forza politica omogenea, interclassista e solida. Così continua De Gasperi, nel racconto di Ossicini:

Per questo non possiamo accettare il ruolo [.... di partito popolare dei soli cattolici avanzati e democratici. Non possiamo più porci come partito riformista, di opposizione; abbiamo bisogno dell'unità politica dei cattolici per avere la possibilità di prendere il potere, perché non esiste alcuna forza in Italia che alla caduta del fascismo possa garantire il passaggio senza traumi dalla situazione a cui il fascismo ci ha portati ad una situazione democratica ${ }^{31}$.

30 A. Ossicini, Cristiani non democristiani, intervista di A. Declich, Editori riuniti, Roma 1980, pp. 39 e sgg.

31 Ivi, pp. 49 e 53. 
Siamo, come detto, nel 1938, l'anno del massimo consenso al fascismo, del varo delle leggi razziali, l'ultimo prima della guerra, ritenuta certa, ma assolutamente incerta nei tempi. È ancora una volta attraverso l'esercizio rigoroso dell'analisi, con la messa a fuoco sia dell'obiettivo da raggiungere, che della strategia operativa ad esso più funzionale, che De Gasperi coglie la vera posta in gioco, ovvero, in questo caso, la necessità di evitare ad ogni costo un vuoto politico dalle conseguenze (come sempre nella storia) catastrofiche. Non c'è in lui alcuna preoccupazione per la continuità formale del partito che ha guidato fino alla sua soppressione per mano fascista - partito che dunque, con occhi del tutto laici, egli considera per quello che è, cioè uno strumento di importanza variabile, a seconda delle situazioni, e chiamato adesso a proseguire la strategia di partenza, rispondendo alla medesima istanza politico-culturale, ma tenendo conto del mutamento del contesto.

Dalle esperienze vissute nel ventennio fascista De Gasperi aveva desunto infatti tre indicazioni fondamentali ${ }^{32}$. La prima era l'esercizio del senso di responsabilità, quello stesso che era mancato al Ppi nella rinuncia all'incarico da parte di Meda nel 1922. La seconda, l'orientamento costante verso una strategia delle alleanze, intesa come antidoto al drammatico errore che, nell' incapacità di allearsi di liberali, socialisti e popolari, aveva consegnato il successo a un movimento assolutamente minoritario in parlamento, come il fascismo. La terza indicazione derivava dall'esperienza vissuta nella fase finale del sistema liberale, le cui deboli istituzioni avevano ceduto troppo facilmente ai colpi delle 'squadracce' fasciste, e che imponeva, per il futuro, di dotare il Paese di istituzioni forti e stabili.

A queste che resteranno tre costanti della visione strategica di De Gasperi, si aggiunge un altro leitmotiv della sua azione politica: il rapporto con la Chiesa. Oggetto degli attacchi di «Civiltà cattolica» e di Pio XI durante la secessione aventiniana, colpito dalle ricadute dell'esilio imposto a Sturzo, sofferente per l'appoggio assicurato dalla Chiesa al regime fascista, il politico popolare aveva sperimentato direttamente i disagi di un difficile dialogo con la gerarchia. $\mathrm{Ma}$ proprio qui stava il problema: senza l'appoggio della Chiesa tutta la strategia volta alla rinascita della democrazia in Italia avrebbe rischiato di saltare, esattamente come era successo quando ci aveva provato il Ppi. E tuttavia De Gasperi, come abbiamo visto, aveva concepito il contributo dei movimenti cattolici al costituzionalismo in genere, e alla costituzione di Weimar nello specifico, in termini d'impegno assolutamente autonomo, il che rendeva l'obiettivo ben poco gradito alla gerarchia. Nell'area più reazionaria del mondo cattolico, in compenso, si dava per scontato, finito il fascismo, che la Chiesa dovesse mantenere un ruolo fondamentale sulla scena politica (al punto da vagheggiare anche per l'Italia un regime di stampo franchista).

Il peso della Chiesa, d'altra parte, sarebbe ulteriormente cresciuto dopo l'8 settembre del 1943, col disgregarsi dell'apparato statale, la fuga del Re, e Pio XII che visitando i romani dopo il bombardamento del quartiere di San Lorenzo, si 
presenta di fatto come una sorta di contraltare istituzionale. Sola istituzione rimasta intatta, con le sue parrocchie diffuse su tutto il territorio nazionale e che diventano subito punti di riferimento non secondari per i civili (e molto spesso anche per la resistenza), la Chiesa appare così una sorta di icona di valori invincibili, su cui poter fare affidamento nel venir meno delle strutture civili. Una rilevanza che si impone anche a livello internazionale, al punto che gli alleati chiedono al Vaticano indicazioni per il post fascismo ricevendone, nel febbraio del 1943, di coerenti con il recente passato: i tre possibili capi del governo suggeriti dal Vaticano sono infatti Vittorio Emanuele Orlando (un vecchio esponente della classe dirigente liberale), Enrico Caviglia (un generale fedele al Re), Luigi Federzoni (il gerarca moderato che avrebbe abbandonato Mussolini il 25 luglio) ${ }^{33}$. E tuttavia, ad onta di queste ombre, la possibilità di far valere le ragioni di un coinvolgimento della Chiesa a difesa della nascente democrazia, rimaneva, per De Gasperi, una sfida fra le più rilevanti del futuro.

\section{Nell'Italia del Comitato di liberazione nazionale}

La sfida che De Gasperi ha davanti, a questo punto, è quella di realizzare la strategia delineata giocando sul condizionamento reciproco fra gerarchia ecclesiastica e mondo cattolico: tentando di conquistare il secondo contando, almeno, sulla non opposizione della prima. I messaggi natalizi del pontefice, sin dal 1941, prendono atto della fase declinante del fascismo e con prudente, ma crescente, consapevolezza, aprono a poco a poco alla prospettiva di una democrazia.

Il mondo cattolico si presenta molto variegato. Luigi Gedda coordina una parte della gerarchia e dell'Azione cattolica, che ben presto prenderà il nome di 'partito romano', sostenendo una strategia di stampo clericale (che, come su ricordato, evoca spesso come riferimento la Spagna franchista). Sul versante opposto, la 'Sinistra cristiana', nuovo nome assunto dal movimento dei Comunisti cattolici, annovera personaggi culturalmente autorevoli (come Felice Balbo, Dino Del Bo, Adriano Ossicini, Franco Rodano, Angelo Romanò, Giorgio Sebregondi) che proprio per la loro levatura erano stati oggetto di attenzione da parte della gerarchia. In Vaticano non veniva infatti escluso che nell'Italia postfascista potesse rivelarsi opportuno contare su presenze cattoliche qualificate in tutti i partiti, quelli estremi compresi.

All'interno delle organizzazioni cattoliche ha un ruolo di grande importanza il Movimento laureati, derivato, come si è accennato più sopra, dall'opera di elaborazione culturale della Fuci (con l'assistenza di monsignor Montini), mentre un ruolo tutt'altro che secondario fu quello del cosiddetto Movimento neoguelfo milanese fondato da Piero Malvestiti, di stampo cattolico liberale e operativo fin

33 P. Blet (éd.), Actes et documents du Saint Siège relatifs à la seconde guerre mondiale, vol. VII. Le Saint Siège et la guerre mondiale. Novembre 1942-décembre 1943, Libreria Editrice Vaticana, Città del Vaticano 1973, p. 364, citato da P. Scoppola, La proposta politica di De Gasperi, il Mulino, Bologna 1977, p. 41. 
dal 1928, molto attivo anche durante la resistenza. Merita sottolineare, all'interno del Movimento laureati, la figura del giovanissimo studioso di economia, dirigente dell'IRI, Sergio Paronetto che, insieme a Saraceno e Vanoni, imposta l'aggiornamento della cultura economica cattolica di fronte alle sfide della modernità. A lui, che scomparirà prematuramente nel marzo del 1945 a soli 34 anni, si deve l'impostazione dell'incontro promosso dal Movimento laureati a Camaldoli (dal quale uscirà l'omonimo Codice, sorta di vademecum per la successiva classe dirigente cattolica, il cui ruolo sarà fondamentale nel processo di rinascita del Paese), con l'intento di fissare una visione sistematica e moderna della politica economica ${ }^{34}$.

Quanto alla posizione della nascente $\mathrm{Dc}$, essa viene sintetizzata in Le idee ricostruttive della Democrazia cristiana, testo preparato da De Gasperi insieme al gruppo di pochi amici del Ppi che, fin dal 1938, si riuniva clandestinamente, e che esce con singolare tempestività il 26 giugno $1943^{35}$.

La 'lunga vigilia' si anima anche, fin dal 1942, dei rapporti clandestini con esponenti delle altre formazioni politiche, che nel gennaio del 1943 porteranno alla costituzione del 'Comitato delle opposizioni antifasciste' (cui De Gasperi partecipa imponendosi all'attenzione degli interlocutori, in particolare di Giorgio Amendola ${ }^{36}$ ), prima forma di coordinamento, per ora soltanto politico, in vista della guerra di liberazione ormai alle porte. Fino al 25 luglio il principale tema di confronto al suo interno sarà il rischio che la caduta di Mussolini, data per imminente nel susseguirsi di rovesci militari, possa dar luogo ad una prosecuzione del regime fascista anche senza il suo leader. All'insediarsi del governo Badoglio, destinato a farsi carico degli effetti del duro armistizio imposto dagli alleati, la proposta di De Gasperi (accettata) sarà di non parteciparvi in prima istanza, per non condividerne la prevedibile sorte, politicamente umiliante. Senza por tempo in mezzo, invece, già il giorno successivo all' 8 settembre, all'annuncio dell'armistizio, il Comitato delle opposizioni si trasforma in 'Comitato centrale di liberazione nazionale' $(\mathrm{Cln})$, col ruolo di strumento immediatamente operativo dell'alleanza politica antifascista.

L'avvio dell'attività del Cln mostra però subito l'esistenza di divergenze interne, che ruotano inizialmente intorno al presidente Ivanoe Bonomi, contrario al progetto di Nenni di far assumere al Cln pieni poteri per abolire la monarchia ${ }^{37}$.

34 T. Torresi, Sergio Paronetto. Intellettuale cattolico e stratega dello sviluppo, il Mulino, Bologna 2017. Oltre a Sergio Paronetto, Pasquale Saraceno ed Ezio Vanoni, del gruppo di Camaldoli facevano parte, fra gli altri, anche Giulio Andreotti, Vittore Branca, Giuseppe Capograssi, Giuseppe Dossetti, Amintore Fanfani, Mario Ferrari Aggradi, Giorgio La Pira, Giuseppe Lazzati, Costantino Mortati, Aldo Moro, Paolo Emilio Taviani.

35 Achille Grandi, Giovanni Gronchi, Attilio Piccioni, Mario Scelba, Giuseppo Spataro, Umberto Tupini, Adone Zoli, con i contributi anche di Pietro Campilli, Mario Cingolani, Camillo Corsanego, Guido Gonella, Bernardo Mattarella, Giulio Pastore, Pier Carlo Restagno.

36 G. Amendola, Lettere a Milano. Ricordi e documenti, 1939-1945, Editori riuniti, Roma 1974, pp. 134-162.

37 Con un ordine del giorno del 16 ottobre (votato in assenza di De Gasperi) il neonato organo politico-operativo delle opposizioni, il Cln, chiede le dimissioni di Badoglio e la formazione di un governo che assuma tutti i poteri dello Stato. Ma già nel gennaio del 1944, al congresso 
In un breve appunto che illustra la sua strategia, De Gasperi fissa in questa circostanza la sua lucida valutazione dei fatti, che conviene riportare integralmente:

dal 16 ottobre ad oggi il metodo rivoluzionario guadagna nella propaganda socialista sempre più terreno contro il procedimento democratico ed evolutivo, onde il presidente (Bonomi) è in pieno diritto di rilevare tale contrasto. Non temo la parola rivoluzione, ne ho piuttosto fastidio, dopo vent'anni che il fascismo, richiamandosi ai diritti della rivoluzione, ha commesso tante soperchierie e violato i diritti dei cittadini, ad ogni modo la vera rivoluzione è la Costituzione. In quanto al gesto insurrezionale, al putsch, sarà permesso di ricordare ai socialisti la tesi di Lenin: «non giocare mai con l'insurrezione e quando si comincia andare fino in fondo»? Volete fare la marcia in Roma amministrata dagli Amgot? O una marcia da Roma in un paese occupato dagli anglosassoni? Comprometterete la causa comune, tanto più che non avete cura di evitare sospetti e in un commento dell'«Avanti» (30 dicembre 1943) schernite i borghesi che ripongono tutte le speranze sugli anglosassoni, mentre gli operai sapranno trovare tuttavia la solidarietà socialista e i vostri amici nel Mezzogiorno tentano di fare lo sciopero simbolico contro Churchill. Ma dove cercherete voi appoggio, in Tito, nella Jugoslavia, contro la quale dovremo difendere palmo a palmo in nostro territorio in Adriatico? Comunque di tali errori non vogliamo essere corresponsabili né lo deve essere il $\mathrm{Cln}^{38}$.

A risolvere la vicenda nel senso auspicato da De Gasperi, spiazzando la linea Nenni, sarà la cosiddetta 'svolta di Salerno' del Pci, annunciata da Palmiro Togliatti il 31 marzo, e il giorno prima anticipata dall'autorevole quotidiano sovietico «Izvestija». Il secondo governo Badoglio riparte così con la partecipazione dei partiti del Cln, vicepresidente del Consiglio il segretario del Pci. Giurerà nelle mani del Re il 12 aprile del 1944, e Winston Churchill si dice convinto che debba restare almeno fino alla liberazione di Roma.

Alla liberazione della capitale, il 4 giugno 1944, grazie alla mediazione di Enrico De Nicola fra casa Savoia e Cln (il cui risultato sarà poi approvato dal Governo militare alleato, l'Amgot), si stabilisce che il Re nomini Umberto luogotenente, con il compito di rappresentare il Paese e conferire l'incarico per la formazione del governo, ma esclusivamente sulla base della unanime designazione del Cln.

Alle dimissioni di Badoglio subentra il governo Bonomi, in crisi già dopo sei mesi, seguito da un secondo governo Bonomi che vivrà fino al 25 aprile, quando arriva il 'vento del Nord'.

di Bari, il Cln attenua la posizione, pur affermando la sua indisponibilità a partecipare a un governo nominato dal Re. Finché, il $1^{\circ}$ marzo, mentre Bonomi presenta un contro documento che delinea un possibile modus vivendi con la monarchia, Nenni richiede l'allontanamento del Re e la proclamazione della repubblica, con Bonomi che subito si dimette da presidente.

38 G. Fanello Marcucci, Alle origini della Democrazia cristiana, 1919-1924, Morcelliana, Brescia 1982. p. 65, citato in P. Craveri, De Gasperi, il Mulino, Bologna 2006, p. 152. 
Del Cln fanno parte, oltre alla Dc, il Pci di Togliatti, impegnato nella costruzione del nuovo partito, il Psiup guidato da Nenni, attestato su posizioni più giacobine, il Partito d'azione, destinato ad esaurire la sua carica in poco più di un quinquennio, il Partito liberale, che vive il travaglio di una trasformazione politica generale che pare spiazzarlo, la Democrazia del lavoro di Bonomi e Meuccio Ruini, destinata a non aver seguito nell'Italia repubblicana. Del quadro politico nazionale farà parte anche, dal dicembre del 1944, il Partito dell"uomo qualunque' di Guglielmo Giannini, che inizia, dandole quel nome, la serie delle formazioni qualunquiste presenti nella successiva storia italiana.

\section{II 'vento del nord' e la fine della guerra}

Dopo la liberazione di Roma la guerra prosegue per altri dieci mesi nelle province centrali e settentrionali, mentre la capitale riprende lentamente la sua attività sotto la sovranità dell'Amgot, che controlla l'intero territorio liberato, nonché il governo.

De Gasperi, già leader del Partito popolare sciolto nel 1926, e che dal 1938 aveva sostanzialmente operato nella clandestinità, all'interno del Cln rappresenta il movimento di area cattolica che, quando il Comitato delle opposizioni decide di ridenominarsi "Comitato delle democrazie" (lui stesso lo racconta in un articolo del 2 febbraio 1944), prende il nome di Democrazia cristiana. La Dc diventerà partito vero e proprio col suo primo congresso, che si tiene a Napoli dal 28 al 29 gennaio 1944, facendo ovviamente riferimento alla sola Italia liberata, cioè alle province meridionali. La prima assemblea del neonato partito si terrà a Roma, al Teatro Brancaccio, il successivo 23 luglio.

Rifiutata l'offerta di Togliatti di assumere presidenza del Consiglio e Ministero degli interni, già con il secondo governo Bonomi De Gasperi assume il Ministero degli esteri (lo manterrà fino all'autunno del 1946), in linea con la convinzione che la priorità dell'Italia sia più che mai, in questo momento, il recupero di una credibilità internazionale, sfida che si rivelerà in effetti fra le più drammatiche del dopoguerra. Fin dai primi incontri ufficiali con gli alleati, il leader cattolico è costretto a prendere atto dello scarso valore riconosciuto alla cobelligeranza italiana (pure apprezzata al momento dell'armistizio) nella guerra di liberazione, e di come l'Italia dovesse darsi parecchio da fare per riconquistare considerazione e stima agli occhi del mondo.

Allo stesso tempo, De Gasperi deve occuparsi della costruzione del partito, organizzarne la presenza nelle aree liberate e governarne le divergenze interne di fronte all'ipotesi di un referendum per la scelta istituzionale. E anche il fronte del Cln va presidiato, alla prova delle difficoltà evidenziate dalla breve durata dei due governi Bonomi, e dal lungo intermezzo della crisi.

Il 25 aprile 1945 l'insurrezione di Milano segna la definitiva liberazione del Paese, e il 'vento del Nord' porta l'eco di chi ha combattuto, più a lungo e vittoriosamente, la guerra partigiana. Ma la fine delle ostilità belliche non restituisce piena sovranità alle forze politiche italiane. Gli alleati continuano infatti a occupare e controllare il territorio italiano, tenendo sotto tutela il governo, mentre il 
Cln deve confrontarsi con un passaggio politico molto delicato: e cioè il transito dalla strategia di ampia solidarietà e di unanimità, che si era resa necessaria nella lotta di liberazione contro il nazi-fascismo, alle inevitabili tensioni suscitate dalle scelte politiche da compiere nella mutata situazione, mentre, nell'euforia della libertà conquistata, emergono le posizioni più giacobine di Nenni $\mathrm{e}$ del Partito d'azione, convinti che si debba affidare il governo alle formazioni partigiane («tutto il potere ai Cln»). Quando, poco dopo il 25 aprile, l'Amgot consente al Cln di recarsi a Milano per incontrare il Cln dell'Alta Italia (Clnai), saranno le notevoli doti di mediatore di De Gasperi a convincere Nenni e gli altri partecipanti della inopportunità di un governo guidato dal leader socialista, nonostante l'assenso già ottenuto sia dal Pci che dal Pd'A.

L'intermezzo del governo di Ferruccio Parri rappresenta il riconoscimento del ruolo svolto fino a quel momento dal movimento resistenziale nella lotta contro il nazifascismo, ma nello stesso tempo anche la presa d'atto della impossibilità di proseguire nella logica dell'unanimismo, più facile da ottenere nei momenti di emergenza, in un'azione di governo chiamata a misurarsi con una fase politica nuova, e obbligata a mettere nel conto la sfida delle mediazioni.

Il 10 dicembre 1945 si insedia il governo De Gasperi, dopo due anni e mezzo (dal 25 luglio del 1943) in cui si erano succeduti cinque governi, intervallati da lunghe crisi, e con tutti i più importanti obiettivi della ripresa ancora inevasi (elezioni amministrative e elezione dell'Assemblea costituente, scelta istituzionale, recupero della sovranità nel Paese, inserimento nell'assetto internazionale, più i drammatici problemi della ricostruzione e della riattivazione delle funzioni essenziali per la vita del Paese), e il territorio nazionale ancora occupato. Ed è proprio sotto la guida dello statista trentino, che resterà ininterrottamente al governo fino al 28 luglio del 1953, che l'Italia entrerà nel dopoguerra. De Gasperi piloterà il Paese verso il ritorno alla democrazia e la scelta repubblicana, attraverso decisioni che si riveleranno fondamentali per la politica interna ed esterna, e che caratterizzeranno i successivi decenni della Repubblica. 